\title{
Ansiedade, depressão e estresse em estudantes universitários: o impacto da COVID-19
}

\author{
Anxiety, depression and stress in university students: \\ the impact of COVID-19
}

\author{
Berta Rodrigues MAIA ${ }^{1}$ ID 0000-0002-6640-6033 \\ Paulo César DIAS ${ }^{1}$ (ID) 0000-0001-6876-3723
}

\section{Resumo}

\begin{abstract}
A pandemia e a inerente alteração de comportamentos, a par da parca previsibilidade, geraram maior ansiedade na população. Nesse sentido, este trabalho teve como objetivo analisar se os níveis de depressão, ansiedade e estresse em estudantes universitários se alteraram no período pandêmico (2020) comparativamente a períodos anteriores/ normais. O estudo foi constituído por dois grupos, sendo a amostra 1 constituída por 460 sujeitos com idade média de 20,14 anos, e a amostra 2 por 159 sujeitos com idade média de 20,40 anos. Todos preencheram um questionário sociodemográfico e as escalas de ansiedade, depressão e estresse. Os estudantes que integraram o estudo no período pandêmico apresentaram níveis significativamente mais elevados de depressão, ansiedade e estresse comparativamente aos que integraram o estudo no período normal. Os resultados sugerem um impacto psicológico negativo da pandemia nos estudantes. Importa continuar a explorar as implicações da pandemia na saúde mental dos estudantes, para que se possam prevenir e minorar os seus efeitos.
\end{abstract}

Palavras-chave: Adulto Jovem; Pandemia; Saúde mental.

\begin{abstract}
The pandemic and the attendant change in behavior, together with the general unpredictability, has generated great anxiety in the population. In this context, the aim of this study was to analyze whether the levels of depression, anxiety and stress in university students have changed during the period of the pandemic (2020) compared with the previous normality. This study comprised two groups, in which sample-1 is composed of 460 subjects with a mean age of 20.14
\end{abstract}

\footnotetext{
$\checkmark \nabla v$

1 Universidade Católica Portuguesa, Centro de Estudos Filosóficos e Humanísticos, Faculdade de Filosofia e Ciências Sociais. Praça da Faculdade, 1, 4710-297, Braga, Portugal. Correspôndencia para/Correspondence to: B.R. MAIA. E-mail: <bmaia@braga.ucp.pt>. Suporte: Trabalho financiado pela Fundação para a Ciência e Tecnologia, no âmbito do Projeto Estratégico do Centro de Estudos Filosóficos e Humanísticos (UID/FIL/00683/2019).

Como citar este artigo/How to cite this article

Maia, B. R., \& Dias, P. C. (2020). Ansiedade, depressão e estresse em estudantes universitários: o impacto da COVID-19. Estudos de Psicologia (Campinas), 37, e200067. http://dx.doi.org/10.1590/1982-0275202037e200067
} 
and sample-2, 159 subjects with a mean age of 20.40. All the participants completed a sociodemographic questionnaire and were assessed by way of the Anxiety, Depression and Stress Scales. The students evaluated during the period of the pandemic presented significantly higher levels of anxiety, depression and stress, compared with students in erstwhile, normal times. Our results suggest that the pandemic has a negative psychological effect on students. It is important to continue exploring the implications of the pandemic on students' mental health, so that its effects can be prevented, or at least mitigated.

Keywords: Young Adult; Pandemics; Mental health.

A doença provocada pelo Coronavirus Disease 2019 (COVID-19) foi identificada pela primeira vez em dezembro de 2019 na cidade de Wuhan, na China, e em janeiro de 2020 a World Health Organization (WHO, 2020a) declarou-a como uma emergência em saúde pública de interesse internacional. Já a esta altura a WHO (2020a) alertava para o fato de esta crise estar gerando estresse na população. Em Portugal, o primeiro caso da COVID-19 foi registrado em 2 de março de 2020. Pouco depois, em 11 de março, a epidemia COVID-19 passou a ser considerada uma pandemia pelo diretor-geral da Organização Mundial de Saúde, Tedros Adhanom Ghenreyesus, uma vez que a doença tinha provocado, até aquela data, mais de 118 mil infectados em 114 países e 4.291 mortes. Em Portugal, em 4 de abril de 2020, estavam registrados 10.524 casos confirmados e 81.087 casos suspeitos, com 266 mortes e 75 pessoas recuperadas.

Todas as pandemias são geradoras de forte impacto social, econômico e político. Basta lembrar, por exemplo, no contexto português, da pandemia de gripe de 1918-1919, conhecida em Portugal por "Pneumônica". Esta revelou ser uma das mais mortíferas, tendo afetado uma em cada três pessoas em nível mundial, o que corresponde a cerca de 500 milhões de pessoas; em Portugal terá sido responsável pela morte de cerca de $2 \%$ da população (Sobral \& Lima, 2018). Se em 1918-1919 a prioridade não assentava em conhecer os efeitos psicológicos da pandemia, em 2020, além de todos os esforços da comunidade científica para se chegar à etiologia e ao tratamento da COVID-19, as respostas à questão têm sido várias e têm implicado áreas muito diversas do conhecimento.

Estudos de revisão publicados recentemente permitem perceber os efeitos da quarentena (Brooks et al., 2020). Tomando-se 24 estudos que envolveram mais de 11 mil residentes ou pessoal médico de áreas afetadas por Middle East Respiratory Syndrome (MERS, Síndrome Respiratória do Oriente Médio), Severe Acute Respiratory Syndrome (SARS, Síndrome Respiratória Aguda Grave), Gripe Suína (H1N1) ou Ébola, observa-se que a maioria deles aponta para efeitos psicológicos negativos, principalmente em termos de confusão, raiva e até estresse pós-traumático. Alguns desses efeitos mantiveram-se num período de tempo mais alargado. Dentre os principais fatores de estresse identificados, sobressaem o efeito da duração do período de quarentena, os receios em relação ao vírus ou à infeção, a frustração, a diminuição de rendimentos, a informação inadequada e o estigma.

Apesar do conhecimento existente, a dimensão da pandemia global é distinta, tal como os seus efeitos na população. Por exemplo, quando comparada com a Influenza (WHO, 2020b), a COVID-19 parece apontar para uma maior taxa de mortalidade, especialmente junto das populações mais velhas ou com comorbidades (Weiss \& Murdoch, 2020; Zhou et al., 2020), e também para uma maior percentagem de infeções severas e críticas, que exigem o apoio de ventilação. Esses fatores levaram os países a tomar medidas sem precedentes, estimando-se que mais de um terço da população global esteja em isolamento, com o fechamento de escolas, universidades e muitas instituições públicas, assim como a instituição do teletrabalho em muitas empresas.

No sentido de minimizar os efeitos da pandemia, a WHO (2020a) tem feito várias recomendações à população geral para que sejam adotados comportamentos e realizadas atividades que permitam reduzir a ansiedade. Do mesmo modo, tem apelado a que se evitem estratégias de coping desajustadas, como o uso de tabaco ou outras substâncias. Também a American Psychological Association (2020) tem disseminado

2 informação e recursos para a população geral e para os técnicos. Em Portugal, a linha do Sistema Nacional 
de Saúde 24 (passou a disponibilizar pela primeira vez aconselhamento psicológico aos portugueses, e a Ordem dos Psicólogos Portugueses criou incentivos à investigação científica em saúde (Ordem dos Psicólogos Portugueses, 2020). Considerando que as alterações rápidas a que os estudantes universitários foram sujeitos, da suspensão das aulas ao decreto do estado de emergência, podem ter desencadeado dificuldades de adaptação e estados emocionais menos positivos, importa explorar as implicações psicológicas dessas circunstâncias.

Que seja do conhecimento dos autores do presente trabalho, poucos estudos exploraram os impactos da COVID-19 e da quarentena sobre a saúde mental de estudantes universitários, principalmente quanto aos níveis de depressão, ansiedade e estresse. No estudo (Wang et al., 2020), realizado com 1.210 participantes de 21 a 30 anos, em 194 cidades na China, 53,8\% da amostra classificaram o impacto psicológico como moderado ou severo, relatando sintomas moderados ou severos de ansiedade (28,8\%), depressão (16,5\%) e estresse $(8,1 \%)$, com diferenças significativas para o sexo feminino. Se é verdade que em outras condições é difícil perceber tais diferenças, estudos anteriores sugerem, consistentemente, maiores indicadores de depressão, ansiedade e estresse entre as mulheres (van de Velde, Bracke, \& Levecque, 2010; Wenjuan, Siqing, \& Xinqiao, 2020). Porém, estudos realizados no período de crise econômica mostraram aumento mais significativo dessa sintomatologia entre os homens (Gili et al., 2016).

Este estudo pretende analisar os níveis de ansiedade, depressão e estresse em estudantes universitários portugueses, usando duas amostras distintas: uma recolhida em 2018 e 2019; e outra recolhida nos oito dias que decorreram entre o período de suspensão das aulas no ensino universitário (10 de março de 2020) e o decreto que instituiu o estado de emergência em Portugal (17 de março de 2020).

\section{Método}

\section{Participantes}

A amostra 1 foi composta por 460 estudantes universitários portugueses, com idade média de 20,14 anos $(D P=1,65$, variação $=18-25)$, em sua maioria solteiros $(99,3 \%, n=455)$ e do sexo feminino $(81,4 \%$, $n=372$ ). Os cursos frequentados eram muito diversos, sendo os mais representados Psicologia (41,0\%, $n=159)$, Ciências da Comunicação $(19,1 \%, n=74)$, Medicina $(M=13,1, n=51)$, Serviço Social $(6,7 \%$, $n=26)$ e Turismo $(7,2 \%, n=28)$.

A amostra 2, por sua vez, foi composta por 159 estudantes universitários portugueses, com idade média de 20,40 anos ( $D P=1,67$, variação $=18-25)$, em sua maioria também do sexo feminino $(77,4 \%, n=123)$ e solteiros (51,1\%, $n=455)$. Os cursos mais representados foram Psicologia (25,2\%, $n=40)$, Enfermagem $(15,1 \%, n=24)$, Ciências da Comunicação $(13,8 \%, n=22)$, Turismo $(11,2 \%, n=21)$ e Geologia $(3,1 \%, n=5)$.

\section{Procedimentos}

Os dados referentes à amostra 1 foram recolhidos em dois momentos distintos: primeiro, em fevereiro/março de 2018 e, depois, em fevereiro/março de 2019, ambas em contexto de sala de aula, em duas universidades portuguesas, uma no norte e outra no centro do país. Os estudantes que aceitaram colaborar completaram os questionários no início do $2^{\circ}$ semestre, evitando assim os períodos de avaliação. Foram obtidos o consentimento informado e garantidos a confidencialidade e o anonimato dos dados recolhidos. A segunda amostra de estudantes universitários foi recolhida através de um questionário online. Também foi obtido o consentimento informado e garantido o anonimato e a confidencialidade dos 
dados.

O questionário foi partilhado com estudantes de universidades de norte a sul do país através do método bola de neve. A recolha dos dados foi feita no espaço de uma semana, entre o período de suspensão das aulas (11 de março de 2020) e o decreto que instituiu o estado de emergência (dia 17 de março de 2020). Em ambas as amostras, os critérios de inclusão foram: ser estudante universitário, ter nacionalidade portuguesa e idade entre 18 e 25 anos.

\section{Instrumentos}

Além de um questionário sociodemográfico contendo questões como idade, estado civil, nacionalidade e curso, os estudantes preencheram a versão portuguesa da Depression, Anxiety and Stress Scales (DASS-21) (Lovibond \& Lovibond, 1995), qual seja, a Escalas de Ansiedade, Depressão e Stress (EADS-21) (Pais-Ribeiro, Honrado, \& Leal, 2004). A EADS-21 é um instrumento de autorresposta composto por três subescalas (ansiedade, depressão e estresse), numa escala tipo Likert variando de 0 ('não se aplicou nada a mim') a 4 ('aplicou-se a mim a maior parte das vezes'). Cada subescala é composta por sete itens. Pontuações mais elevadas na EADS-21 correspondem a níveis mais elevados de ansiedade, depressão e estresse. A validação portuguesa revelou boas propriedades psicométricas, com alfas de Cronbach a variar de 0,74 (ansiedade) a 0,85 (depressão). No presente estudo, foram encontrados alfas de Cronbach's de 0,94 para a subescala da depressão, de 0,93 para a subescala de ansiedade, e de 0,92 para a subescala de estresse.

\section{Análise de dados}

Trata-se de um estudo não experimental, transversal, quantitativo e descritivo, com duas amostras de conveniência. Os dados foram analisados recorrendo ao Software IBM ${ }^{\circledR}$ SPSS ${ }^{\circledR}$ (versão 26). Foi analisada a consistência interna da EADS-21, determinando os coeficientes de alfa de Cronbach, considerando-se os valores entre 0,65 e 0,70 como aceitáveis, 0,70 e 0,80 como bons, e 0,90 como muito bons. Para a descrição da amostra calcularam-se estatísticas descritivas. Como análise univariada, foram determinadas frequências absolutas ( $n$ ) e relativas (\%) para as variáveis qualitativas. Na análise bivariada, para as variáveis quantitativas, foi utilizado o teste $t$ de Student para amostras independentes. Finalmente, para uma avaliação do tamanho de efeito, foi utilizado o $d$ de Cohen. A amostra 1 foi recolhida no âmbito de um estudo aprovado pela Comissão de Ética da Faculdade de Medicina da Universidade de Coimbra, Portugal (ref. CE-026/2018).

\section{Resultados}

A análise das respostas aos itens da EADS-21 (Tabela 1) permite perceber que no período pandêmico há itens cuja pontuação, relativamente às três subescalas - depressão, ansiedade e estresse - é substancialmente mais elevada do que a obtida no período normal (e.g., itens 2, 5, 10, 13, 15, 18, 19, 20 e 21).

Foi conduzido um teste $t$ de Student para amostras independentes para comparar os níveis de ansiedade, depressão e estresse em função do sexo dos sujeitos. Em ambos os períodos (normal e pandêmico), o sexo masculino apresentou médias mais elevadas de depressão (Tabela 2), porém sem diferenças significativas ( $p=0,810$ e $p=0,948$, respectivamente). Já no sexo feminino, em ambos os períodos as médias foram mais elevadas nas subescalas de ansiedade e de estresse, sendo as diferenças não significativas $(p=0,181$; $p=0,096$ e $p=0,580 ; p=0,658$, respectivamente)

Finalmente, foi conduzido um teste $t$ de Student para amostras independentes com o objetivo de comparar os níveis de ansiedade, depressão e estresse nos períodos normal e pandêmico. Quando comparado 
Tabela 1

Análise descritivas dos itens da EADS-21. Período normal (Norte e Centro de Portugal, 2018/2019) e Período pandêmico (Portugal, 2020)

1 de 2

\begin{tabular}{|c|c|c|c|c|c|c|c|c|c|c|c|c|c|c|c|c|}
\hline \multirow{3}{*}{ EADS-21 } & \multicolumn{8}{|c|}{ Período normal } & \multicolumn{8}{|c|}{ Período pandêmico } \\
\hline & \multicolumn{2}{|c|}{1} & \multicolumn{2}{|c|}{2} & \multicolumn{2}{|r|}{3} & \multicolumn{2}{|r|}{4} & \multicolumn{2}{|c|}{1} & \multicolumn{2}{|c|}{2} & \multicolumn{2}{|c|}{3} & \multicolumn{2}{|r|}{4} \\
\hline & $n$ & $(\%)$ & $n$ & (\%) & $n$ & $(\%)$ & $n$ & $(\%)$ & $n$ & (\%) & $n$ & $(\%)$ & $n$ & (\%) & $n$ & (\%) \\
\hline $\begin{array}{l}\text { 1. Tive dificuldades } \\
\text { em me acalmar }\end{array}$ & 175 & $(38,0)$ & 206 & $(44,8)$ & 65 & $(14,1)$ & 14 & $(3,0)$ & 59 & $(37,1)$ & 74 & $(46,5)$ & 16 & $(10,1)$ & 10 & $(6,3)$ \\
\hline $\begin{array}{l}\text { 2. Senti a minha } \\
\text { boca seca }\end{array}$ & 254 & $(55,2)$ & 162 & $(35,2)$ & 37 & $(8,0)$ & 7 & $(1,5)$ & 62 & $(39,0)$ & 57 & $(35,8)$ & 25 & $(15,7)$ & 15 & $(9,4)$ \\
\hline $\begin{array}{l}\text { 3. Não consegui } \\
\text { sentir nenhum } \\
\text { sentimento po- } \\
\text { sitivo }\end{array}$ & 312 & $(67,8)$ & 113 & $(24,6)$ & 32 & $(7,0)$ & 3 & $(0,7)$ & 84 & $(52,8)$ & 55 & $(34,6)$ & 11 & $(6,9)$ & 9 & $(5,7)$ \\
\hline $\begin{array}{l}\text { 4. Senti dificulda- } \\
\text { des em respirar }\end{array}$ & 325 & $(70,7)$ & 107 & $(23,3)$ & 25 & $(5,4)$ & 2 & $(0,4)$ & 90 & $(56,6)$ & 54 & $(34,0)$ & 10 & $(6,3)$ & 5 & $(3,1)$ \\
\hline $\begin{array}{l}\text { 5. Tive dificulda- } \\
\text { des em tomar } \\
\text { a iniciativa para } \\
\text { fazer coisas }\end{array}$ & 200 & $(43,5)$ & 188 & $(40,9)$ & 60 & $(13,0)$ & 11 & $(2,4)$ & 42 & $(26,4)$ & 70 & $(44,0)$ & 37 & $(23,3)$ & 10 & $(6,3)$ \\
\hline $\begin{array}{l}\text { 6. Tive tendência } \\
\text { a reagir em } \\
\text { demasia em } \\
\text { determinadas } \\
\text { situações }\end{array}$ & 180 & $(39,1)$ & 185 & $(40,2)$ & 80 & $(17,4)$ & 15 & $(3,3)$ & 52 & $(32,7)$ & 71 & $(44,7)$ & 25 & $(15,7)$ & 11 & $(6,9)$ \\
\hline $\begin{array}{l}\text { 7. Senti tremores } \\
\text { (por exemplo, } \\
\text { nas mãos) }\end{array}$ & 314 & $(68,3)$ & 88 & $(19,1)$ & 47 & $(10,2)$ & 11 & $(2,4)$ & 95 & $(59,7)$ & 31 & $(19,5)$ & 24 & $(15,1)$ & 9 & $(5,7)$ \\
\hline $\begin{array}{l}\text { 8. Senti que es- } \\
\text { tava a utilizar } \\
\text { muita energia } \\
\text { nervosa }\end{array}$ & 205 & $(44,6)$ & 154 & $(33,5)$ & 80 & $(17,4)$ & 21 & $(4,5)$ & 68 & $(42,8)$ & 53 & $(33,3)$ & 27 & $(17,0)$ & 11 & $(6,9)$ \\
\hline $\begin{array}{l}\text { 10. Senti que não } \\
\text { tinha nada a es- } \\
\text { perar do futuro }\end{array}$ & 320 & $(69,6)$ & 99 & $(21,5)$ & 32 & $(7,0)$ & 9 & $(1,9)$ & 85 & $(53,5)$ & 25 & $(22,0)$ & 20 & $(12,6)$ & 19 & $(11,9)$ \\
\hline $\begin{array}{l}\text { 11. Dei por mim a } \\
\text { ficar agitado }\end{array}$ & 180 & $(43,5)$ & 198 & $(37,4)$ & 79 & $(14,9)$ & 21 & $(4,0)$ & 52 & $(32,7)$ & 67 & $(42,1)$ & 25 & $(15,7)$ & 15 & $(9,4)$ \\
\hline $\begin{array}{l}\text { 12. Senti dificul- } \\
\text { dade em me } \\
\text { relaxar }\end{array}$ & 159 & $(34,6)$ & 209 & $(45,4)$ & 56 & $(12,2)$ & 15 & $(3,5)$ & 48 & $(30,2)$ & 63 & $(39,6)$ & 28 & $(17,6)$ & 20 & $(12,6)$ \\
\hline $\begin{array}{l}\text { 13. Senti-me desa- } \\
\text { nimado e me- } \\
\text { lancólico }\end{array}$ & 185 & $(40,2)$ & 203 & $(44,1)$ & 60 & $(11,4)$ & 17 & $(3,2)$ & 46 & $(28,9)$ & 62 & $(39,0)$ & 37 & $(23,3)$ & 14 & $(8,8)$ \\
\hline $\begin{array}{l}\text { 14. Estive intoleran- } \\
\text { te em relação a } \\
\text { qualquer coisa } \\
\text { que me impe- } \\
\text { disse de termi- } \\
\text { nar aquilo que } \\
\text { estava a fazer }\end{array}$ & 253 & $(55,0)$ & 151 & $(32,8)$ & 52 & $(11,3)$ & 4 & $(0,9)$ & 64 & $(40,3)$ & 61 & $(38,4)$ & 23 & $(14,5)$ & 11 & $(6,9)$ \\
\hline $\begin{array}{l}\text { 15. Senti-me qua- } \\
\text { se a entrar em } \\
\text { pânico }\end{array}$ & 331 & $(72,0)$ & 83 & $(18,0)$ & 35 & $(7,6)$ & 11 & $(2,4)$ & 88 & $(55,3)$ & 39 & $(24,5)$ & 22 & $(13,8)$ & 10 & $(6,3)$ \\
\hline $\begin{array}{l}\text { 16. Não fui capaz } \\
\text { de ter entusias- } \\
\text { mo por nada }\end{array}$ & 327 & $(71,1)$ & 99 & $(21,5)$ & 25 & $(5,4)$ & 9 & $(2,0)$ & 93 & $(58,5)$ & 42 & $(26,4)$ & 16 & $(10,1)$ & 8 & $(5,0)$ \\
\hline
\end{tabular}


Tabela 1

Análise descritivas dos itens da EADS-21. Período normal (Norte e Centro de Portugal, 2018/2019) e Período pandêmico (Portugal, 2020)

2 de 2

\begin{tabular}{|c|c|c|c|c|c|c|c|c|c|c|c|c|c|c|c|c|}
\hline \multirow{3}{*}{ EADS-21 } & \multicolumn{8}{|c|}{ Período normal } & \multicolumn{8}{|c|}{ Período pandêmico } \\
\hline & \multicolumn{2}{|c|}{1} & \multicolumn{2}{|c|}{2} & \multicolumn{2}{|r|}{3} & \multicolumn{2}{|c|}{4} & \multicolumn{2}{|r|}{1} & \multicolumn{2}{|c|}{2} & \multicolumn{2}{|c|}{3} & \multicolumn{2}{|r|}{4} \\
\hline & $n$ & $(\%)$ & $n$ & $(\%)$ & $n$ & $(\%)$ & $n$ & (\%) & $n$ & (\%) & $n$ & $(\%)$ & $n$ & $(\%)$ & $n$ & (\%) \\
\hline $\begin{array}{l}\text { 17. Senti que não ti- } \\
\text { nha muito valor } \\
\text { como pessoa }\end{array}$ & 345 & $(75,0)$ & 82 & $(17,8)$ & 23 & $(5,0)$ & 10 & $(2,2)$ & 90 & $(56,6)$ & 36 & $(22,6)$ & 23 & $(14,5)$ & 10 & $(6,3)$ \\
\hline $\begin{array}{l}\text { 18. Senti que por } \\
\text { vezes estava } \\
\text { sensível }\end{array}$ & 101 & $(22,0)$ & 242 & $(52,6)$ & 93 & $(20,2)$ & 24 & $(5,2)$ & 31 & $(20,1)$ & 59 & $(37,1)$ & 45 & $(28,3)$ & 23 & $(14,5)$ \\
\hline $\begin{array}{l}\text { 19. Senti alterações } \\
\text { no meu cora- } \\
\text { ção sem fazer } \\
\text { exercício }\end{array}$ & 306 & $(66,5)$ & 105 & $(22,8)$ & 44 & $(9,6)$ & 5 & $(1,1)$ & 69 & $(43,4)$ & 55 & $(34,6)$ & 28 & $(17,6)$ & 7 & $(4,4)$ \\
\hline $\begin{array}{l}\text { 20. Senti-me assus- } \\
\text { tado sem ter } \\
\text { tido uma boa } \\
\text { razão para isso }\end{array}$ & 318 & $(69,1)$ & 110 & $(23,9)$ & 24 & $(5,2)$ & 8 & $(1,7)$ & 79 & $(49,7)$ & 52 & $(32,7)$ & 21 & $(13,2)$ & 7 & $(4,4)$ \\
\hline $\begin{array}{l}\text { 21. Senti que a mi- } \\
\text { nha vida não } \\
\text { tinha sentido }\end{array}$ & 372 & $(80,9)$ & 56 & $(12,2)$ & 24 & $(5,2)$ & 8 & $(1,7)$ & 97 & $(61,0)$ & 36 & $(22,6)$ & 12 & $(7,5)$ & 14 & $(8,8)$ \\
\hline
\end{tabular}

Nota: 1: não se aplicou nada a mim; 2: aplicou-se a mim algumas vezes; 3: aplicou-se a mim muitas vezes; 4: aplicou-se a mim a maior parte das vezes. EADS-21: Escalas de Ansiedade, Depressão e Stress.

Tabela 2

Pontuações médias na EADS-2. Período normal (Norte e Centro de Portugal, 2018/2019) e Período pandêmico (Portugal, 2020)

\begin{tabular}{|c|c|c|c|c|c|c|c|c|}
\hline \multirow{3}{*}{ Subescalas EADS-21 } & \multicolumn{4}{|c|}{ Período normal } & \multicolumn{4}{|c|}{ Período pandêmico } \\
\hline & \multicolumn{2}{|c|}{ Mulheres } & \multicolumn{2}{|c|}{ Homens } & \multicolumn{2}{|c|}{ Mulheres } & \multicolumn{2}{|c|}{ Homens } \\
\hline & M & $D P$ & M & $D P$ & M & $D P$ & $M$ & $D P$ \\
\hline Ansiedade & 3,31 & $(3,67)$ & 2,74 & $(2,94)$ & 12,41 & $(4,91)$ & 11,92 & $(4,09)$ \\
\hline Depressão & 3,39 & $(3,75)$ & 3,49 & $(3,46)$ & 12,67 & $(5,20)$ & 12,61 & $(5,05)$ \\
\hline Estresse & 6,11 & $(4,50)$ & 5,22 & $(4,13)$ & 14,20 & $(4,92)$ & 13,78 & $(5,14)$ \\
\hline
\end{tabular}

Nota: EADS-21: Escalas de Ansiedade, Depressão e Stress; M: Média; DP: Desvio Padrão.

o período normal ao pandêmico, foram encontradas diferenças estatisticamente significativas nos níveis de depressão $(M=3,39, D P=3,69$ versus $M=12,66, D P=5,15), t(617)=-24,51, p=<0,001$; nos níveis de ansiedade $(M=3,19, D P=3,54$ versus $M=12,30, D P=4,73), t(617)=-25,51, p=<0,001$; e nos níveis de estresse $(M=5,94, D P=4,43$ versus $M=14,10, D P=4,95), t(617)=-19,42, p=<0,001$. Ainda assim, a magnitude das diferenças foi baixa para as três subescalas: Cohen's $d=0,207$ (depressão), 0,218 (ansiedade) e 0,173 (estresse).

\section{Discussão}

Este estudo teve como objetivo explorar os níveis de ansiedade, depressão e estresse em estudantes universitários portugueses, comparando dois momentos distintos, isto é, um período normal (2018 e 2019) e o período pandêmico (entre a suspensão das aulas e a decretação do estado de emergência em Portugal).

Os resultados confirmam um aumento significativo de perturbação psicológica (ansiedade, depressão e estresse) entre os estudantes universitários no período pandêmico comparativamente a períodos normais. Esses resultados vão ao encontro de outros estudos internacionais que analisaram o efeito psicológico da COVID-19 e de outras pandemias (Wang et al., 2020; Weiss \& Murdoch, 2020). As informações transmitidas 
pelos diversos meios, bem como a discussão que se colocou socialmente a propósito das medidas de confinamento, podem ter contribuído para o aumento das pontuações médias. Apesar de, naquela altura, ainda não serem contabilizados os óbitos, o acompanhamento da situação em nível global e o aumento dos casos positivos para COVID-19 parecem ter gerado níveis de ansiedade, depressão e estresse entre os estudantes universitários, mesmo se sabendo que este não seria um grupo de maior risco em termos de letalidade (Weiss \& Murdoch, 2020; Zhou et al., 2020).

A natureza global e a informação existente, talvez mais que as diferenças culturais, podem ajudar a explicar a indistinção entre sexos encontrada em estudos anteriores (Weiss \& Murdoch, 2020). Se a literatura internacional tende a encontrar diferenças entre os grupos, os dados apresentados sugerem efeitos semelhantes na amostra estudada. Estudos posteriores, especialmente de natureza longitudinal, poderão ajudar a perceber melhor essas tendências.

O uso de duas amostras distintas, ambas de conveniência, é a maior limitação deste estudo, não sendo, por isso, possível extrapolar os resultados. Do mesmo modo, não se pode assumir que a elevação nos níveis de ansiedade, depressão e estresse se deva apenas à pandemia. Ela pode ser devida a vários outros factores (e.g., personalidade, apoio social percebido). Ainda assim, este estudo é um ponto de partida para a investigação futura nesta área.

Em suma, este estudo realça, pelos seus resultados, a necessidade de estar-se atento aos efeitos psicológicos desta pandemia, para que as respostas subsequentes, no âmbito da saúde mental, possam ser oportunamente asseguradas.

\section{Conclusão}

Os resultados deste estudo sugerem que esta pandemia provoca efeitos deletérios na saúde mental dos estudantes universitários, reforçando que importa continuar a investigar o tema, para que se possam perceber os mecanismos e reações psicológicas subjacentes a um período de vida tão atípico e desafiante.

Existindo dados de que esses efeitos podem prolongar-se no tempo, importa traçar estratégias de prevenção ou remediação. Num trabalho conjunto de diversas instituições públicas, desde a Organização Mundial da Saúde à Associação Americana de Psicologia ou, em Portugal, da Ordem dos Médicos à Ordem dos Psicólogos Portugueses, têm sido emitidas recomendações no sentido da normalização e validação de sentimentos de tristeza, ansiedade ou confusão geradas pelas informações transmitidas pela mídia. Do mesmo modo, tem-se apelado à manutenção de estilos de vida saudáveis, à manutenção de redes sociais de apoio através das tecnologias de informação e comunicação, e também a uma postura mais criativa ou de mobilização de recursos ou estratégias anteriores para lidar com situações adversas. Num momento posterior, certamente será necessário aprofundar a discussão e implementar programas de promoção de competências sociais e emocionais junto de populações mais jovens, bem como estratégias de remediação para episódios traumáticos decorrentes desta pandemia.

\section{Colaboradores}

B. R. MAIA foi responsável pela concepção e desenho do estudo, pelo processo de recolha de dados, pela análise estatística, pela redação do artigo (todas as secções) e pela revisão e aprovação da versão final do artigo. P. C. DIAS participou na redação do artigo (Introdução, Discussão e Conclusão) e na revisão e aprovação da versão final do artigo.

\section{Referências}

American Psychological Association. (2020). Pandemics COVID-19: general resources. Retrieved from https://www.apa. org/practice/programs/dmhi/research-information/pandemics 
Brooks, S. K., Webster, R. K., Smith, L. E., Woodland, L., Wessely, S., Greenberg, N., \& Rubin G. J. (2020). The psychological impact of quarantine and how to reduce it: rapid review of the evidence. The Lancet, 395(10227), 912-920. http:// dx.doi.org/10.1016/S0140-6736(20)30460-8

Gili, M., López-Navarro, E., Castro, A., Homar, C., Navarro, C., García-Toro, M., ... Roca, M. (2016). Gender differences in mental health during the economic crisis. Psicothema, 28(4), 407-413. http://dx.doi.org/10.7334/psicothema2015.288

Lovibond, P., \& Lovibond, S. (1995). The structure of negative emotional states: comparison of the depression anxiety stress scales (DASS) with the Beck Depression and Anxiety Inventories. Behavioral Research and Therapy, 33(3), 335-343. http://dx.doi.org/10.1016/005-7967(94)00075-u

Ordem dos Psicólogos Portugueses. (2020). Linha SNS24 passa a disponibilizar pela primeira vez aconselhamento psicológico aos portugueses. Recuperado de https://www.ordemdospsicologos.pt/pt/noticia/2769

Pais-Ribeiro, J. L., Honrado, A., \& Leal, I. (2004). Contribuição para o estudo da adaptação portuguesa das escalas de ansiedade, depressão e stress (EADS) de 21 itens de Lovibond e Lovibond. Psicologia, Saúde e Doenças, 5(2), 229-239. http://www.scielo.mec.pt/pdf/psd/v5n2/v5n2a07.pdf

Sobral, J. M., \& Lima, M. L. (2018). A epidemia da pneumónica em Portugal no seu tempo histórico. Ler História, 73, 45-66. http://dx.doi.org/lerhistoria.4036

van de Velde, S., Bracke, P., \& Levecque, K. (2010). Gender differences in depression in 23 European countries: cross-national variation in the gender gap in depression. Social Science and Medicine, 71(2), 305-313. http://dx.doi. org/10-1016/j.socsmed.2010.03.035

Wang, C., Pan, R., Wan, X., Tan, Y., Xu, L., \& Ho, C. (2020). Immediate psychological responses and associated factors during the initial stage of the 2019 Coronavirus Disease (COVID-19) epidemic among the general population in China. International Journal of Environmental Research and Public Health, 17(5), 1729. http://dx.doi.org/10.3390/ ljerph17051729

Weiss, P., \& Murdoch, D. R. (2020). Clinical course and mortality risk of severe COVID-19. The Lancet, 395(1022), 1014-1015. http://dx.doi.org/10.1016/S0140-6736(20)30633

Wenjuan, G., Siqing, P., \& Xinqiao, L. (2020). Gender differences in depression, anxiety, and stress among college students: a longitudinal study from China. Journal of Affective Disorders, 263(15), 292-300. http://dx.doi.org/10.1016/ jad.2019.11.121

World Health Organization. (2020a). Mental health and psychosocial considerations during the COVID-19 outbreak. Geneva: Author. Retrieved from https://www.who.int/docs/default-source/coronaviruse/mental-health-considerations. pdf

World Health Organization. (2020b). Coronavirus disease 2019: situation Report-46. Geneva: Author. Retrieved from https://www.who.int/docs/default-source/coronaviruse/situation-reports/20200306-sitrep-46-covid-19. pdf?sfvrsn=96b04adf_2

Zhou, F., Yu, T., Du, R., Fan, G., Liu, Y., Liu, Z., ... Cao, Bin. (2020). Clinical course and risk factors for mortality of adult inpatients with COVID-19 in Wuhan, China: a retrospective cohort study. The Lancet, 395(10229), 1054-1062. http:// dx.doi.org/10.1016/S0140-6736(20)30566-3

Recebido: abril, 13, 2020

Aprovado: abril 23, 2020 